\title{
Psiquiatría de enlace en servicios médico-quirúrgicos de dos hospitales de Santiago
}

\author{
G uillermo Hernández $\mathrm{G}^{1}$, Paola G allardo-Zurita ${ }^{1}$, \\ Rafael Sepúlveda J ${ }^{2}$, Pilar Morales de Sétien $\mathbf{G}^{2}$. \\ Liaison psychiatry in two public \\ hospitals of Santiago
}

Background: Medical and surgical teams consult psychiatrists when abnormal behavior or psychopathological traits are observed in their patients. Aim: To describe the experience of liaison psychiatry teams in public hospitals. Material and methods: Analysis of 128 consultations, involving 110 patients. Eighty eight patients were subjected to the structured Clinical Interview for DSM-IV-TR Axis I Disorders - Patient Edition, the Mini Mental State score and to a search for personality disorders. Results: The most commonly consulted problems were depression in 44 patients, substance abuse in 29 and suicidal behavior in 21 . The psychiatric study detected that $8 \%$ of patients were asymptomatic, $8 \%$ had stress reactions, $16.5 \%$ had substance abuse, $14.6 \%$ had alcohol abuse, $11 \%$ had a major depression, 10\% had delirium or dementia, $9 \%$ had anxiety disorders, $4 \%$ schizophrenia, 18\% personality disorders and $19 \%$ had suicidal behaviors. Conclusions: Psychiatric consultations in medical and surgical services are common and have a wide variety of severity (Rev Méd Chile 2004; 132: 725-32).

(Key Words: Alcohol-related disorders; Community psychiatry; Depressive disorder; Mental disorders; Substance abuse)

Recibido el 5 de septiembre, 2003. Aceptado en versión corregida el 1 de abril, 2004.

${ }^{1}$ Departamento de Psiquiatría y Salud Mental Sur, Facultad de Medicina, Universidad de Chile. ${ }^{2}$ Servicio de Psiquiatría, Hospital Barros Luco Trudeau.

L

a investigación sobre prevalencia de trastornos psiquiátricos en pacientes de servicios médicoquirúrgicos da cifras de 20 a $62 \% 1-5$. La coexistencia de síntomas o trastornos psiquiátricos y enfermedades médicas ha demostrado ser una condición que afecta la evolución y el pronóstico de los enfermos ${ }^{6-9}$, el costo de las hospitalizaciones y el tiempo de estadía hospitalaria10-14. Varios autores afirman que la comorbilidad médicopsiquiátrica está subdiagnosticada, porque espontáneamente se solicita asistencia psiquiátrica para 0,7-10\% de los potenciales pacientes ${ }^{1,3,13,15,16}$.

Correspondencia a: Dr. Guillermo Hernández G. Departamento de Psiquiatría Sur, Universidad de Chile. Casilla 226, Correo 13 San Miguel, Santiago. Fono Fax: 5517906.

E-mail: ghernandez@machi.med.uchile.cl
Los problemas de interfase médica-psiquiátrica, por su frecuencia y efectos, han estimulado al hospital general a destacar profesionales o a formar unidades especializadas en abordarlos ${ }^{2}$.

Una investigación multicéntrica de equipos de consultoría de enlace y medicina psicosomática en Europa (56 servicios, 11 países), informó que los equipos médico-quirúrgicos piden atención para pacientes con síntomas psiquiátricos o con quejas somáticas inexplicables $(57,2 \%)$, que se han autoinferido lesiones (17\%), que son abusadores de sustancias (7\%) o con dificultades para afrontar la enfermedad médica $(4,1 \%)^{15,18,19}$.

El estudio psiquiátrico de los pacientes $(\mathrm{N}=14.717$; CIE 10) encontró sin trastornos a 10,8\%, $24,6 \%$ con trastornos neuróticos; del humor 19,5\%; 
psicoorgánicos 18,4\%; por abuso de sustancias $15,5 \%$; esquizofrenias y esquizotípicos $4,4 \%$; trastornos de personalidad $4 \%$; y eran cuadros somatomorfos y relacionados con estrés 2,2\%15,18,19.

Investigaciones nacionales sobre interconsultoría psiquiátrica en hospitales generales encontraron cuadros reactivos y neuróticos (43 y 44,9\%), desórdenes de la personalidad (35\%), cuadros orgánicos (30 y 10\%), psicosis (18 y 8\%), alcoholismo (3,3 y 8\%) y desórdenes psicofisiológicos y sexuales ${ }^{20-22}$.

Con este estudio quisimos conocer qué problemas originan la demanda espontánea actual de los servicios médico-quirúrgicos a psiquiatría, describir los hallazgos del psiquiatra interconsultor a esa demanda y discutir sucintamente las posibilidades de la psiquiatría de enlace en los hospitales donde se hizo el estudio.

\section{MATERIAL Y MÉTODO}

El Servicio de Psiquiatría del Hospital Barros LucoTrudeau (HBLT) presta asistencia psiquiátrica ambulatoria, con internamiento y de urgencia. Pacientes hospitalizados en servicios médico-quirúrgicos y en el hospital de enfermedades infecciosas (HLC), se atienden cuando sus tratantes lo solicitan con una «hoja de interconsulta».

A 128 solicitudes recibidas en el servicio los miércoles y viernes del semestre octubre-2002 marzo-2003 por motivos asistenciales, se sumaron objetivos de investigación. Para cumplirlos, se registró la información sobre origen, fechas de emisión, recepción y respuestas; datos clínicos, psicológicos, sociales o de cualquier otro orden aportados por los consultantes en esas solicitudes.

La información referida a diagnósticos psiquiátricos, problemas o conductas que causaron las solicitudes, se recogió en los términos usados por el consultante, excepto que inequívocamente los expresara mejor algún concepto psiquiátrico, por ejemplo, incumplimiento terapéutico, afrontamiento de enfermedad, agitación psicomotora.

Se excluyeron síntomas, conductas o descripciones usados para fundamentar el diagnóstico psiquiátrico de derivación y datos adicionales al problema o situación que se pedía evaluar.

Pacientes y estudio psiquiátrico. Las 128 solicitudes identificaban a 110 pacientes. Se tomó contac- to con 96. A 88 se les efectuó completo el estudio psiquiátrico diseñado. Este consistió en una evaluación multiaxial de cinco ejes.

I. Trastornos mentales: se obtuvieron administrando la versión para pacientes psiquiátricos de la «ntrevista Clínica Psiquiátrica Estructurada para el DSM IV-TR» SCID I/P23,24. Los trastornos no incluidos en este instrumento se diagnosticaron de acuerdo a criterios específicos del DSM IV-TR.

Para objetivar rendimientos cognitivos y amnésicos dudosos en pacientes con sospecha de trastornos del grupo delirium, demencias, trastornos amnésicos u otros trastomos cognoscitivos se usó el Mini Mental State Exam (MMSE) ${ }^{25,26 .}$

II. Trastornos de la personalidad y retraso mental: se evaluaron usando los criterios clínicos específicos del DSM IV-TR.

III. Enfermedades médicas: los diagnósticos médicos fueron proporcionados por los consultantes.

IV. Problemas psicosociales y ambientales: se discriminaron con la información de la encuesta abierta inicial y de la gráfica vital de la SCID I/P.

V. Evaluación de la actividad global del paciente: resultó de aplicar la escala de puntajes destinada a medir el efecto de la enfermedad mental sobre la actividad psicosocial, social y laboral incluida en la SCID I/P. Excluye limitaciones ocasionadas por causas físicas y ambientales.

La SCID I/P es un instrumento clínico extenso con detalladas instrucciones para su uso.

Se inicia con una entrevista abierta que recoge información biodemográfica, antecedentes psicopatológicos, psicopatología durante el mes anterior y evalúa el funcionamiento social actual, el contexto ambiental y posibles desencadenantes de la psicopatología actual. Con estos datos se formulan hipótesis de diagnósticos psiquiátricos actuales más probables y los que deben descartarse. Una «gráfica vital» complementa la información psicopatológica y la ordena en una línea temporal.

Módulos específicos exploran la presencia o ausencia de síntomas y comportamientos que exige el DSM IV-TR para diagnosticar trastornos del estado de ánimo, esquizofrenia y otros trastornos psicóticos; trastornos relacionados con sustancias, de ansiedad, somatomorfos, de la conducta alimentaria y adaptativos actuales o de curso de vida. El DSM IV-TR tiene códigos compatibles con la CIE $10^{29}$. 
Los datos obtenidos fueron analizados con el programa EpiInfo 2002.

La información referente a los ejes III y V no fue incluida en este reporte.

\section{RESULTADOS}

La Tabla 1 muestra el origen de las solicitudes. El $82,7 \%$ provino del hospital general. Medicina intema solicitó al psiquiatra más que cirugía y maternidad con $22,7,17,3$ y 13,6\%, respectivamente. Los otros nueve servicios acumularon $29 \%$ de las solicitudes del hospital general. Los consultantes calificaron $20,4 \%$ de sus solicitudes como urgencias.

Los consultores respondieron a $70 \%$ de las solicitudes el día que se recibieron, $14 \%$ dentro de 48 h, 5\% al tercer día y 4-7 días después de su emisión, 10\%.

El 76,5\% de los pacientes tuvo una visita y 23,5\% entre dos y seis.

Las características biodemográficas de los pacientes se describen en la Tabla 2.

Predominaron levemente las mujeres. El promedio de edad para ambos géneros fue próximo a los 40 años, con rangos similares para ambos géneros. Menos de la mitad estaban casados. Un tercio tenía enseñanza básica o sin educación. Más de dos tercios no tenía actividad laboral. Un tercio tenía como fuente de ingresos económicos su trabajo o

Tabla 1. Servicios de origen de las solicitudes a Psiquiatría de Enlace para 110 pacientes

\begin{tabular}{|clcr|}
\hline & Servicio & Frecuencia & Porcentaje \\
\hline HBLT & Medicina & 25 & 22,7 \\
& Cirugía & 19 & 17,3 \\
& Maternidad & 15 & 13,6 \\
& Neurología & 6 & 5,5 \\
& Traumatología & 6 & 5,5 \\
& Ginecología & 5 & 4,5 \\
& UTI & 5 & 4,5 \\
& Urología & 3 & 2,7 \\
& UCI & 1 & 0,9 \\
& Otorrinolaringología & 1 & 0,9 \\
& Neurocirugía & 1 & 0,9 \\
Otros & 4 & 3,6 \\
HLC & Salas de HLC & 19 & 17,3 \\
Total & Total & 110 & 10 \\
\hline
\end{tabular}

HBLT: Hospital Barros Luco-Trudeau

HLC: Hospital Lucio Córdova el de su cónyuge. Un tercio vivía solo, allegados a no familiares, en la calle o en instituciones.

Los motivos para solicitar intervención del psiquiatra se describen y cuantifican con cifras absolutas en la Tabla 3. En orden decreciente fueron problemas del estado de ánimo, por uso de sustancias, alcohol 0 ambos, por ideación 0

Tabla 2. D atos biodemográficos de 110 enfermos médico-quirúrgicos con consulta a Psiquiatría de Enlace

\begin{tabular}{|lr|}
\hline Variable & $\%$ \\
\hline Género & \\
Mujeres & \\
Edad (años) & 57,3 \\
$\quad<49$ & \\
$50-69$ & 70 \\
$>70$ & 23,6 \\
Estado civil & 6,4 \\
Casado & \\
Soltero & 45,4 \\
Separados & 20 \\
Viudos & 8,2 \\
DNC* & 4,5 \\
Escolaridad (años) & 20,9 \\
0-4 & \\
5-8 & 16,4 \\
9-12 & 17,3 \\
Más de 12 & 38,2 \\
DNC* & 6,3 \\
Actividad laboral actual & 21,8 \\
No & \\
DNC* & 71,8 \\
Ingreso económico principal & 20,9 \\
De trabajo propio 0 de cónyuge \\
Pensionados por enfermedad física, \\
mental o viudez & 34,5 \\
Dependientes de padres o parientes & 11,8 \\
Jubilación & 6,4 \\
Mendicidad & 0,9 \\
Otros (rentas, subsidios, & 10 \\
ahorros, indemnizaciones) & \\
DNC* & 22,7 \\
Vive con & \\
Familia & 66,4 \\
Solo & 11,8 \\
Otro & 21,8 \\
\hline D : Dato no consgnador \\
\hline
\end{tabular}

* DNC : Dato no consignado. 
intento de suicidio, por trastornos psicóticos, por problemas relacionados con embarazo o puerperio, por agitación psicomotora o delirium; por ansiedad. También se pidió al psiquiatra traslado de pacientes a psiquiatría, procedimientos terapéuticos o apoyo a pacientes con síntomas emocionales; evaluación de pacientes que no adherían a tratamientos médicos o afrontaban mal la enfermedad médica, con problemas de la personalidad o retraso mental, a candidatos a trasplante de órgano o cirugía de la obesidad, con problemas psicosociales o VIH+; para diagnóstico diferencial y a pacientes agresivos o con insomnio.
$\mathrm{Al}$ efectuar la visita para evaluación, 20\% de los pacientes no estuvo disponible para el estudio; 11 mujeres y 2 hombres habían egresado por alta; una mujer había fallecido. Dos pacientes estaban sedados con fármacos prescritos, dos intoxicados con fármacos autoadministrados, dos con delirium severos. Dos no fueron investigados por otras causas.

El estudio psiquiátrico completo se efectuó a 88 pacientes. El tiempo empleado en cada paciente dependió de su calidad como informante y de la complejidad de sus problemas. En minutos fue de $10-30$ para $16,3 \%$; $31-59$ para $24,5 \%$; $60-90$ para $48 \%$ y de $91-120$ y más para $8 \%$.

\section{Tabla 3. M otivos planteados a Psiquiatría de Enlace en solicitudes de evaluación a 110 pacientes hospitalizados en servicios médico-quirúrgicos*}

\begin{tabular}{|lr|}
\hline Motivos para solicitar consulta a psiquiatría & Frecuencia \\
\hline Depresión/estado depresivo/síndrome depresivo & 44 \\
Enfermedad/trastomo/depresión bipolar & 4 \\
Drogadicción/adicción/poliadicción/abuso/consumo/deprivación de sustancias & 11 \\
Abuso/consumo/dependencia a sustancias y alcohol & 3 \\
Alcoholismo crónico/delirium tremens/síndrome de deprivación a alcohol & 11 \\
Detener ingesta/manejo de alcoholismo/derivación a Unidad de Dependencias & 3 \\
Tabaquismo & 1 \\
Intentos de suicidio & 21 \\
Ideación suicida & 3 \\
Crisis de pánico/angustia/síndrome ansioso-depresivo/angustia-depresión & 10 \\
Tratamiento de duelo perinatal & 4 \\
Hiperemesis gravídica/embarazo no deseado/malformación fetal & 7 \\
Evaluación de competencia materna & 2 \\
Evaluación pretrasplante de órgano/precirugía de obesidad & 6 \\
Esquizofrenia/psicosis crónica/psicopatía alucinatoria catatónica/psicosis postictal & 9 \\
Oligofrenia/retardo mental & 3 \\
Abandono familiar/violencia intrafamiliar/quiebre familiar/conflicto familiar & 4 \\
Efecto emocional/conductual de hospitalización/afrontamiento de enfermedad médica & 3 \\
Agitación psicomotora/síndrome delirioso/síndrome confusional & 11 \\
Psicosis esteroidal/prevención de efectos psiquiátricos del uso de corticoides & 6 \\
Síndrome convulsivo psicógeno/diagnóstico diferencial de convulsiones/síndrome conversivo & 3 \\
Apoyo uso/manejo/psicofármacos (sedantes/antidepresivos) & 5 \\
Paciente VIH (+) & 4 \\
Abandono/incumplimiento de tratamiento médico & 5 \\
Solicitud de hospitalización en psiquiatría & 3 \\
Trastorno/alteración de personalidad & 4 \\
Psicoterapia/psicoterapia de apoyo & 2 \\
Paciente agresivo & 3 \\
Otros & 3 \\
\hline
\end{tabular}

*Hay solicitudes con 2 o más motivos. 
La Tabla 4 recoge los resultados del estudio psiquiátrico.

Nueve enfermos no tenían síntomas ni antecedentes psicopatológicos, $10 \%$. Nueve tenían síntomas emocionales causados por estresores de su ambiente natural, la enfermedad o la hospitalización y no calificaron para trastornos mentales.

Los trastornos de ansiedad alcanzaron a $28 \%$. En este grupo 17\% son fobias específicas, que en el escenario médico importan sólo si son a agujas, sangre, daño.
La dependencia actual a sustancias ilícitas: cannabis, cocaína y sustancias múltiples alcanzó a $18 \%$, sin diferencias estadísticas entre hombres y mujeres. La dependencia actual a alcohol afectó a $12,8 \%$ con predominio significativo en hombres (Fisher=0,01). El abuso de alcohol afectó a 1,8\% de la muestra y se dio sólo en hombres. Dependencia a benzodiacepinas se encontró en 1,1\%.

Los cuadros depresivos mayores en curso, sumaron $13,5 \%$ para hombres y mujeres con predominio no significativo de mujeres. La depre-

Tabla 4. Condiciones y trastomos mentales actuales (D SM IV TR) diagnosticados por Psiquiatras de Enlace en 88 pacientes hospitalizados en servicios médico-quirúrgicos*

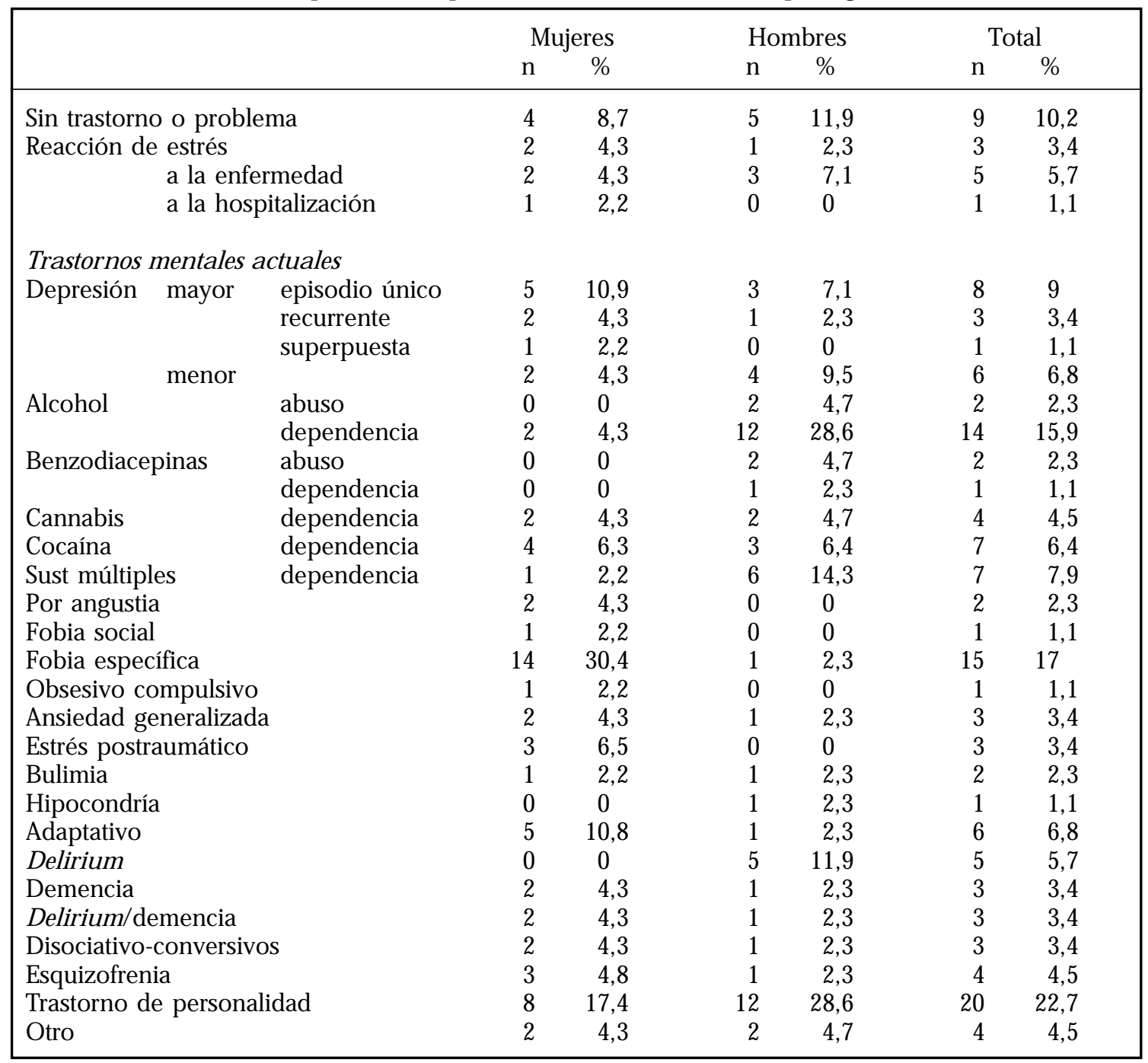

* Hay pacientes con dos o más trastornos. 
sión menor afectó a 6,8\% y predominó en los hombres. Demencias, delirium y delirium/demencias afectaron a $12,5 \%$. Los trastornos adaptativos afectaron a $6,8 \%$.

El 4,5\% de los pacientes presentaban esquizofrenias y $3,4 \%$ calificaron para trastornos disociativos-conversivos. Un hombre $(1,1 \%)$, presentaba un cuadro hipocondríaco.

Trastornos de la personalidad, sin considerar la forma específica del trastorno, encontramos en $22 \%$.

El $42 \%$ de los pacientes presentaba dos a cuatro cuadros psiquiátricos comórbidos.

El número total de diagnósticos psiquiátricos fue 114 y el promedio por paciente 1,4.

Los cuadros y condiciones psiquiátricas de Curso de Vida se muestran en la Tabla 5. Se trata de trastornos que habiendo estado presentes en algún momento de la vida del paciente, en la evaluación actual carecen de importancia para las acciones médicas de los clínicos a cargo. La depresión mayor de curso de vida fue significativamente mayor en mujeres que en hombres (test exacto de Fisher $=0,01$ ). La dependencia y el abuso de curso de vida a alcohol afectaron sólo a hombres.

Suicidalidad: Comprometía a 21 pacientes, 12 mujeres y nueve hombres. Tres habían sido hospi- talizados por lesiones autoinferidas con arma cortante o de fuego; 14 se habían intoxicado con fármacos sedantes, antidepresivos, anticonvulsivantes u opioides; dos habían ingerido ácidos 0 tóxicos; uno había intentado el colgamiento, uno se había lanzado desde altura. Nueve fueron dados de alta antes de la visita del psiquiatra. Entre los evaluados 11 eran casos con diagnóstico en varios ejes. En la historia psiquiátrica se detectó que en ellos interactuaban problemas psicosociales como del núcleo primario de apoyo en ocho pacientes; de vivienda, económicos, del ambiente de residencia en cinco; había trastornos de la personalidad en siete y dos tenían la condición VIH+. En cinco pacientes, el cuadro psiquiátrico hallado, eje I, fue depresión, en tres, dependencia a alcohol, en dos, problemas de la conducta alimentaria y uno era dependiente a sustancias múltiples.

\section{DisCUSIÓN}

La asistencia psiquiátrica a pacientes en servicios médico-quirúrgicos de los hospitales BLT y LC se efectúa de modo constante desde hace 25 años. En comparación con los resultados de un estudio anterior en los mismos escenarios ${ }^{22}$, la utilización del servicio de enlace psiquiátrico se ha multiplicado por tres. Medicina interna acumulaba casi

Tabla 5. Trastornos mentales de curso de vida (D SM IV TR) diagnosticados por Psiquiatras de Enlace en 88 pacientes hospitalizados en servicios médico-quirúrgicos

\begin{tabular}{|lccccccc|}
\hline \multirow{2}{*}{ Trastornos de curso de vida } & \multicolumn{2}{c}{ Mujeres } & \multicolumn{2}{c}{ Hombres } & \multicolumn{2}{c|}{ Total } \\
& $\mathrm{n}$ & $\%$ & $\mathrm{n}$ & $\%$ & $\mathrm{n}$ & $\%$ \\
\hline \multirow{2}{*}{ Depresión mayor } & 13 & 28,2 & 2 & 4,7 & 15 & 17 \\
Alcohol abuso & 0 & 0 & 3 & 7,1 & 3 & 3,4 \\
$\quad$ dependencia & 0 & 0 & 5 & 11,9 & 5 & 5,7 \\
Benzodiacepinas abuso & 0 & 0 & 3 & 7,1 & 3 & 3,4 \\
Cannabis abuso & 1 & 2,1 & 0 & 0 & 1 & 1,1 \\
Estimulantes dependencia & 1 & 2,1 & 2 & 4,7 & 3 & 3,4 \\
Opioides abuso & 0 & 0 & 1 & 2,3 & 1 & 1,1 \\
Cocaína abuso & 0 & 0 & 1 & 2,3 & 1 & 1,1 \\
Sustancias múltiples dependencia & 1 & 2,1 & 1 & 2,3 & 2 & 2,3 \\
ASHTA & 1 & 2,1 & 0 & 0 & 1 & 1,1 \\
Fobia social & 1 & 2,1 & 1 & 2,3 & 2 & 2,3 \\
Fobia específica & 2 & 4,3 & 0 & 0 & 2 & 2,3 \\
\hline
\end{tabular}


$2 / 3$ de las solicitudes, hoy con $22 \%$, está poco por encima de cirugía. Maternidad, neurología, traumatología, la UTI y el hospital de enfermedades infecciosas han aumentado sus solicitudes.

Los pacientes de psiquiatría de enlace eran mayoritariamente mujeres, actualmente son hombres y mujeres casi en igual proporción, menores de 49 años la mayoría y de bajo nivel socioeconómico y educacional.

Los problemas por los que se requiere al psiquiatra son múltiples. El listado obtenido en nuestro estudio se asemeja a lo descrito en la literatura internacional actual ${ }^{1,15,18,19}$ y como es de esperar, se diferencia de los datos nacionales publicados en la década 1980-8920-22. Representa los problemas psiquiátricos de alta prevalencia en la comunidad como depresión, trastornos por consumo de alcohol y sustancias, ansiedad y concentra solicitudes para pacientes hospitalizados por conductas suicidas, 19\%. También muestra el interés de los tratantes porque se les apoye en aspectos psicosociales, comportamientos ante la enfermedad y en la terapéutica psiquiátrica de sus pacientes. Los temas introducidos por psiquiatras en la última década en la maternidad del HBLT, han sido acogidos por obstetras y matronas y motivan consultas específicas ${ }^{28}$. Se solicita también que el psiquiatra ofrezca apoyo preventivo con evaluaciones antes de trasplante de órganos o uso de corticoides.

En sus respuestas, el psiquiatra hace visitas en que no encuentra destinatarios; a pacientes que no están en condiciones de una evaluación psiquiátrica tradicional, y a pacientes sin psicopatología, estos correspondieron a 36\% de los 110 pacientes identificados por sus tratantes para evaluación psiquiátrica. Entre los pacientes estudiados y con trastornos psiquiátricos, los de la personalidad resultaron los más frecuentes aunque menos que 35\%, hallado en 1981 por Florenzano $^{20}$, y muy por sobre $4 \%$, comunicado en el estudio europeo $15,18,19$. Sigue en frecuencia, la dependencia a sustancias y muy cercanamente la dependencia a alcohol. Sumados alcanzan a 34\% de todos los trastornos diagnosticados. La asociación de estos trastornos a violencia explicaría el aumento de consultas a psiquiatría de enlace desde traumatología. La depresión mayor actual es un problema de magnitud importante. Asociada a otros trastornos, como de la personalidad o por uso de alcohol y sustancias, explicaría la alta frecuencia de suicidalidad. Los delirium-demencias, también frecuentes tienen entre sus causas la dependencia a alcohol. Los trastornos de ansiedad mortifican a un grupo importante de pacientes.

Resulta interesante verificar que varios problemas que constituyen motivos frecuentes de consulta a psiquiatría de enlace, encuentran correlato en los cuadros psiquiátricos hallados.

Esto habla de un trabajo de interfase médicopsiquiátrico bien encaminado.

Algunos aspectos inmediatos pueden mejorarse. Por ejemplo, la población que no pudo evaluarse podría rescatarse difundiendo entre los consultantes adecuadas recomendaciones referentes a la emisión de las solicitudes y agilizando el manejo administrativo de la derivación. Un trabajo en red y más recursos profesionales podrían dar mejores respuestas para aquellos pacientes que necesitan traslado a unidades psiquiátricas o seguimiento.

Varios de los items del listado de solicitudes hechas a psiquiatría constituyen un desafío para una unidad de psiquiatría de enlace y plantean exigencias a las tres tareas que se han definido para ésta, que algunos califican como subespecialidad de la psiquiatría: asistencia a pacientes, educación de médicos y cirujanos en aspectos básicos de la psiquiatría e investigación en temas de interfase médico-psiquiátrica. Los resultados aquí expuestos muestran que estamos cumpliendo, en alguna medida, esos propósitos. Los programas de psiquiatría de consultoría de enlace al hacerse cargo oportuna y adecuadamente de la población con comorbididad psiquiátrica ahorrarían recursos. Ello ha difundido la actividad desde la que ejercen unidades de alta especialización dedicadas $24 \mathrm{~h}$ diarias a estos pacientes, hasta las que efectúan psiquiatras que conservan responsabilidades profesionales preferentes en unidades psiquiátricas dedicadas a otros temas. La psiquiatría de enlace en nuestro medio podría señalarse como en posición intermedia. 


\section{REFERENCIAS}

1. Bronheim H, Fulop G, Kunkel E, Muskin P, Schindier B, Yates W et AL. The Academy of Psychosomatics Medicine Practice Guidelines for Psychiatric Consultation in the General Medical Setting. Psychosomatics 1998; 39: S8-S30.

2. DiefENBACher A. Implementation of a Psychiatric Consultation Service. A single site observational study over a 1 year period. Psychosomatics 2001; 42: 404-10.

3. Hernández G, ibáñez C, Kimelman M, Oreilana G, Montino O, NúÑEZ C. Prevalencia de trastomos psiquiátricos en hombres y mujeres hospitalizados en un Servicio de Medicina Intema de un hospital de Santiago de Chile. Rev Méd Chile 2001; 129: 1279-88.

4. Hernández G, Montino O, Kimelman M, Oreliana G, NúNEZ C, IbÁÑEZ C. Prevalencia de trastomos psiquiátricos por uso de alcohol y otras sustancias en hombres y mujeres hospitalizados en medicina interna de un hospital de Santiago de Chile. Rev Méd Chile 2002; 130: 651-60.

5. Tapia P, Michel C, Koppman A. Morbilidad Psiquiátrica en un Hospital General. Rev Psiquiatr Clin 1994; 31: 99-107.

6. Frasure-Smith N, Lespérance F, Talajic M. Depression following myocardial infarction. Impact on six months survival. JAMA 1993; 270: 1819-25.

7. Frasure-Smith N, Lespérance F, Talajic M. Depression and 18 months prognosis after myocardial infarction. Circulation 1995; 91: 999-1005.

8. Micheison D, Stratakis C, Him L, Reynolds J, Gauven E, Chrousos G et al. Bone Mineral Density in women with depression. N Engl J Med 1996; 335: 1176-81.

9. KorNFELD D. Consultation-Liaison Psychiatry: Contributions to medical practice. Am J Psychiatry 2002; 159: 1964-72.

10. Furlanetto L, Da Silva R, Bueno J. The impact of psychiatry comorbidity on lenght of medical inpatients. Gen Hosp Psychiatry 2003; 25: 14-9.

11. Hosaka T, Aoki T, Watanabe T, Okuyama T, Kurosawa H. Comorbidity of depression among physically ill patient and its effects on the lenght of hospital stay. Psychiatry Clin Neurosci 1999; 53: 491-5.

12. Creed F, Morgan R, Fiddier M, Marshall S, Guthrie E, House A. Depression and anxiety impair HealthRelated Quality of life and are associated with increased cost in general medical inpatients. Psychosomatics 2002; 43: 302-9.

13. Strain J, Lyons J, Hammer J, Fahs M, Levobitz A, PadDison P ET aL. Cost offset from psychiatric consultation liaison intervention with elderly hip fracture patients. Am J Psychiatry 1991; 148: 1044-9.
14. Hosaka T, Aoki T, Watanabe T, Okuyama T, Kurowa $H$. General hospital from the perspective of medical economics. Psychiatry Clin Neurosci 1999; 53: 449-53.

15. Huyse F, Herzog T, Lobo A, Malt U, Opmeer B, Stein B ET AL. Consultation Liaison Psichiatry service delivery: results from a European study. Gen Hosp Psychiatry 2001; 23: 124-32.

16. Gala C, Rigateli M, De Bertolini C, Rupolo G, GABRIELI F, GRASSI L A multicenter investigation of consultation-liaison psychiatry in Italy. Gen Hosp Psychiatry 1999; 21: 310-7.

17. Holmes A, Judd F, Lloyd J, Dakis J, Cairns F, KerR L, Kiang M, McKinnon J. A contemporary view of psychiatry services in a general medical hospital. Australasian Psychiatry 2001; 9: 243-7.

18. Huyse F, Herzog T, Lobo A, Malt U, Opmeer B, Stein B ET AL. European consultation liaison services and their user populations: The European consultation liaison workgroup collaborative study. Psychosomatics 2000; 41: 330-8.

19. Huyse F, Herzog T, Lobo A, Malt U, Opmeer B, Stein B ET AL. European Consultation Liaison Psychiatric services the ECLW collaborative study. Acta Psychiatr Scand 2000; 101: 360-6.

20. Florenzano R. Interconsultas psiquiátricas en un Hospital General. Rev Méd Chile 1981; 109: 661-7.

21. Eva P, JeREZ S, VARAS Y. Revisión de interconsultas psiquiátricas. Rev Psiquiat Clin 1984; 21: 39-46.

22. HeRNÁNDEZ G, LóPEZ L Psiquiatría y Medicina en el Hospital General. Estudio de la Interconsulta Psiquiátrica. Rev Chil Neuro-Psiquiat 1985; 23: 197-204.

23. First M, SPITZER R, Gibbon M, Wiшiam J. Estructured Clinical Interview for DSM-IV-TR Axis I Disorders Patient Edition (SCID-I/P, 2/2001 revision) Biometrics Research Departament, New York Psychiatric Institute, 1051 Riverside Drive - Unit 60, New York, New York 10032

24. DSM-IV-TR Manual Diagnóstico y Estadístico de los Trastornos Mentales Texto Revisado. Barcelona, España 2002.

25. Cassem N, Murray G. Pacientes con delirios. En: Cassem N. Massachusetts General Hospital. Manual de Psiquiatría en Hospitales Generales; pp 105-127 Hartcourt Brace de España 1998.

26. FaLK W, Mariyn A. Pacientes dementes. En: Cassem N. Massachusetts General Hospital. Manual de Psiquiatría en Hospitales Generales; pp 129-154. Hartcourt Brace de España 1998.

27. OMS. CIE 10. Trastornos mentales y del comportamiento. Meditor, Madrid 1992.

28. Hernández G, Kimelman M, Montino O. Salud mental perinatal en la asistencia hospitalaria del parto y puerperio. Rev Méd Chile 2000; 128: 1283-9. 